\title{
¿Cómo enfrentar a las burocracias sindicales? Algunas estrategias democratizadoras en los 80
}

\author{
Mónica Gordillo
}

IDH - CONICET - UNC

\section{Introducción}

La dictadura de 1976 representó un punto de inflexión en el ámbito laboral por diversas razones. En primer lugar por la crudeza de la represión hacia el movimiento obrero que en esta ocasión aplicó la metodología de terrorismo de Estado. Ello introdujo como novedad el exilio de militantes sindicales que crearon redes con el exterior, generando experiencias de resistencia articulada. Además, en el marco de la crisis del fordismo de mediados de los años 70 , el contacto con otras experiencias y la formación sindical en contextos diferentes dotarian de recursos y aprendizajes para plantear estrategias frente a los cambios que comenzaban a operarse en el mundo del trabajo.

En segundo lugar, en sintonía con el objetivo refundacional del Proceso de Reorganización Nacional (PRN), fue el período más largo de intervención a los sindicatos y de suspensión de la normativa laboral, intentándose su reemplazo por una nueva forma de organización basada en la ley de asociaciones profesionales (LAP) de 1979.

Una tercera diferencia importante con las anteriores dictaduras fue que la suspensión de la legalidad laboral por parte de la última fue aprovechada por los sectores sindicales que habian enfrentado cuestionamientos desde sus bases para fortalecerse en el nuevo contexto, contando para ello con el apoyo del gobierno militar que buscaba generar sus propias bases de sustentación.

De este modo, los desafios para la recuperación de los sindicatos debieron orientarse en diferentes sentidos. En primer lugar, en recomponer la representación en los lugares de trabajo de donde habian sido barridos los militantes más combativos; por otro lado, enfrentar a la vez tanto las amenazas y limitaciones impuestas por el gobierno militar como a las dirigencias que se habian consolidado en los gremios. Otro desafio 
era, además, recuperar las organizaciones en un contexto económico crítico donde al mismo tiempo se colocaba a la democracia como marco de sentido y horizonte que obligaba a buscar nuevas formas de acción diferentes de las más radicalizadas del pasado.

Para comenzar, se hace necesario entonces registrar brevemente algunos antecedentes sobre los intentos de organización para enfrentar a la dictadura, que sirvieron de base para la constitución de redes mantenidas luego en democracia. La bibliografia sobre oposición sindical en ese período no es muy abundante todavia, aunque sí se cuenta con algunos trabajos pioneros (Falcón, 1982; Pozzi, 1988; Bitrán y Schneider, 1992) que se concentraron principalmente en los conflictos y protestas desarrollados en esos años. Otra línea de indagación consideró la conformación de distintos nucleamientos sindicales dentro de lo que podría considerarse como un análisis de las relaciones entre el gobierno y los sindicatos (Mc Guire, 1997; Palomino, 2005). Sin embargo, lo que me interesa destacar en esta ocasión es la constitución de algunas agrupaciones internas opositoras a las conducciones sindicales y redes de oposición, primero a la dictadura y luego para competir por la normalización de los gremios, utilizando los resquicios que se fueron abriendo a partir de la crisis del régimen militar.

Efectivamente, algunos investigadores destacaron el importante papel cumplido por el sindicalismo para promover la salida de las dictaduras en Argentina, Brasil y Uruguay (Collier Berins, 1991, 1999; Munck, 1989, entre otros) y la aparición de un nuevo tipo de sindicalismo, surgido durante la dictadura en Brasil y fortalecido ya en democracia, como el que se expresó en la conformación del Partido de los Trabajadores (PT) y de la Central Única de Trabajadores (CUT) (Moreira Alves, 1984; Godio, 2003); también señalaron la renovación producida en el sindicalismo uruguayo a partir de la creación del Plenario Intersindical de los Trabajadores (PIT). En cambio, en Argentina, si bien numerosas investigaciones se ocuparon de las relaciones de los sindicatos con el gobierno democrático de Alfonsin y de las transformaciones operadas dentro del peronismo, casi no existen trabajos que indaguen el surgimiento de propuestas tendientes a la democratización dentro de algunos sectores sindicales. Por el contrario, a partir del restablecimiento del régimen democrático, la reivindicación de la democracia sindical pareció quedar en manos exclusivas del gobierno de Alfonsín, quien con su proyecto de Ley de Reordenamiento Sindical -conocido como Proyecto Mucci- personificó ese ideal, apareciendo su derrota en el Senado en el mes de marzo de 1984 como la derrota de la reivindicación misma. Esto limitó la posibilidad de indagar otros intentos en esa dirección planteados por sectores que habian recuperado protagonismo politico con esas banderas. 
El análisis de algunas de esas propuestas y estrategias antiburocráticas y democratizadoras es el objeto de este artículo. Me propongo abordar las planteadas por sectores sindicales que frente a la salida democrática de 1983 recuperaron tradiciones combativas para enfrentar tanto a la dictadura como a las dirigencias gremiales asociadas con ese pasado, impulsando propuestas democratizadoras dentro del marco general de revalorización de la legalidad democrática. Presentaré algunos ejemplos de los logros conseguidos por esos sectores en las primeras elecciones sindicales con el objeto de reflexionar sobre cómo se entendía la democratización sindical en el contexto de los años 80, valorando por último algunos avances en esa dirección. El análisis no pretende una reconstrucción exhaustiva de las experiencias sino, más bien, ofrecer algunas claves teórico-metodológicas y avances empíricos para analizar la democratización y contribuir a pensar su contraparte, es decir la burocratización sindical.

\section{¿Qué hacer frente a la dictadura y sus aliados en el mundo sindical?}

En primer lugar habria que considerar quiénes representaban a la burocracia sindical en el diagnóstico de los sectores opositores. Ella era la que había apoyado la centralización y fortalecimiento del verticalismo -contenido por ejemplo en la LAP de 1973-, la que había intervenido directamente o aceptado la intervención de los gremios combativos entre 1974-1976 y, durante la dictadura, desafiliado a los trabajadores que planteaban posiciones contrarias al gobierno además de proveer los cuadros para las pocas actividades sindicales desarrolladas durante el régimen, por ejemplo en la representación del país en las reuniones anuales de la Organización Internacional del Trabajo (OIT). Dentro de esa definición sólo un sector era reivindicado, el conocido como grupo "de los 25", formado a comienzos de 1977 con los gremios que habían mantenido sus autoridades electas antes del golpe, dado que -más allá de la heterogeneidad y diferencias ideológicas- eran los únicos con conducciones legitimas, lo que convertía al sector en el núcleo desde donde empezar a plantear la recuperación sindical.

La resistencia sindical comenzó a poco de ocurrido el golpe, combinando acciones internas con redes de apoyo desde el exterior. Una de esas iniciativas fue la propiciada por Eduardo Luis Duhalde desde su exilio en Madrid al conformar el PROA (Partido Revolucionario de los Obreros Argentinos), integrado por militantes peronistas, marxistas y de la izquierda nacional. Haroldo Logiurato, dirigente de ATE La Plata y uno de los fundadores de la Juventud Peronista (JP) en esa ciudad, fue uno de los dos secretarios generales; sin embargo la organización 
se desarticuló tras su muerte junto a otros militantes en manos del Ejército en junio de 1977 (Chaves, 2015: 150).

Otra red para proteger y brindar apoyo económico a militantes sindicales que provenian de tradiciones combativas y que comenzaron a exiliarse desde 1975 fue la promovida por Raimundo Ongaro, dirigente gráfico y ex secretario general de la Confederación General del Trabajo (CGT) de los Argentinos, quien con apoyo de la Confederación General Democrática del Trabajo francesa, ligada a la Central Mundial del Trabajo (CMT), organizó el Centro Sindical con personas de la Secretaría de Relaciones Internacionales de la Federación Gráfica Bonaerense (FGB) en 1977 y el nucleamiento Trabajadores y Sindicalistas Argentinos en el Exilio, reunido por primera vez en París en 1978. Por su parte, la propia Central Latinoamericana de Trabajadores (CLAT) a través de la Universidad de los Trabajadores de América Latina (UTAL), dio asilo y trabajo en Venezuela a otros dirigentes de primera línea de la FGB también exiliados con Ongaro, como fue el caso de Alicia Fondevilla (Basualdo: 2006).

A su vez una red paralela fue creada por el Movimiento Peronista Montonero (MPM) que, desde 1978, decidió coordinar la resistencia sindical a la dictadura. Los encargados eran Armando Croatto y Gonzalo Chaves, quienes regresaron del exilio con esta tarea. Cuando se exilió en 1977 Chaves era miembro de la mesa nacional de la Juventud Trabajadora Peronista (JTP) (Chaves, 2015: 219).

También a fines de 1977 se conformó otro núcleo con fuerte conexiones con la CLAT, que brindó apoyo para la constitución de una agrupación opositora en el seno de la Asociación de Trabajadores del Estado (ATE). La dictadura había prorrogado el mandato vigente de Horvath como secretario general, sin embargo el verticalismo que caracterizó a su gestión generó disconformidad y enfrentamiento interno, incluso en el seno del Consejo Directivo Central. El distanciamiento de algunos dirigentes que lo componían, como Héctor Quagliaro, Carlos Custer, Víctor De Gennaro y Germán Abdala fue correspondido con sus expulsiones del sindicato por inconducta. Estos miembros, estimulados por el liderazgo de Quagliaro, dieron forma a la Agrupación Nacional Unidad y Solidaridad de ATE (ANUSATE), creada en diciembre de 1977 cuando se reunieron durante tres días en la Casa de Nazareth de la Iglesia de la Santa Cruz, trabajadores estatales prescindidos y expulsados con la intención de conformar una agrupación interna de oposición al gobierno dictatorial y a la conducción de Horvath. En ella participaron representantes de nueve seccionales, se reunieron dirigentes con trayectorias importantes quienes en su mayoria habian integrado la CGT de los Argentinos.

Luego de constituida, ANUSATE emprendió una intensa campaña 
de difusión de su propuesta en las seccionales del interior a través de giras personales, de su periódico y de la participación en el Instituto de Formación y Capacitación Social (INFORCCAS) que formaba parte del Consejo Coordinador Argentino Sindical (CCAS), adherido a la CLAT. A su vez De Gennaro llegó a presidir el INFORCCAS y en 1982 lo hizo Germán Abdala; de este modo se estrechaba la conexión entre el CCAS y ANUSATE así como con la CLAT.

A partir de esos diferentes núcleos de oposición pero tomando como base para la acción legal al grupo "de los 25", el año 1979 fue escenario de acciones conjuntas como la convocatoria al paro general del 27 de abril, luego del cual los principales dirigentes del sector fueron detenidos por ocho meses (Chaves, 2015: 212). Una vez conocido el proyecto de ley de asociaciones profesionales del gobierno militar, ANUSATE promovió el Plan Nacional de Acción dispuesto para noviembre de 1979 que se planteó como un encuentro nacional de distintos sectores sociales. En lo que refiere específicamente a la acción sindical, llamaba a la movilización de los trabajadores, a la realización de asambleas en los lugares de trabajo y en los sindicatos, campañas de difusión en la prensa, entre otras acciones para culminar con un plebiscito nacional en los lugares de trabajo a los efectos de fijar la posición frente a la nueva LAP y a la ley de obras sociales. ${ }^{1}$

Para entonces también el sector sindical ligado al MPM comenzó a editar de manera clandestina el periódico Confluencia Sindical donde, como lo plantea el título de la publicación, la intención era privilegiar el interés de clase por sobre las divisiones ideológicas, colocando como prioridad la reconstrucción de la trama sindical desde las bases con el objetivo de lograr la unidad y la normalización. Ese periódico fue continuado a partir de 1983 con otra publicación, El Diecisiete, pero ya como vocero de las Agrupaciones Sindicales Peronistas (ASP) que se constituyeron para entonces (Chaves, 2015: 235).

En efecto, el escenario cambió luego de la derrota en la guerra de Malvinas. A partir de la segunda mitad de 1982, en el marco de salida del régimen, la mayoría de los dirigentes sindicales que se encontraban presos fueron liberados. Otros lo habían sido con anterioridad, pero para entonces con la condición de irse del país. Este había sido el caso de los metalúrgicos de la seccional de Villa Constitución en la provincia de Santa Fe, portadores de una experiencia antiburocrática en los 70 , tales

1. Asociación de Trabajadores del Estado. Agrupación Nacional Unidad y Solidaridad, noviembre-diciembre de 1979, p. 3 Llama la atención la promoción del plebiscito como instrumento para la expresión de la voluntad soberana, dada la poca tradición que éste tenía en el país. Podría pensarse en la difusión de ciertos marcos de sentido que trascendian las fronteras locales, teniendo en cuenta que al año siguiente éste se utilizó en Uruguay para oponerse a las medidas de la dictadura. 
como Pascual D’Errico y Ángel Porcu, miembros de la Comisión Interna de Acindar, Zenón Sánchez y Victorio Paulón del comité de lucha de marzo de 1975, presos de la dictadura hasta 1978. Por su parte Alberto Piccinini, principal referente de ese movimiento, obtuvo la libertad en 1981 y, luego de un viaje al exterior, regresó al país con la intención de reorganizar a los trabajadores. Ésta será otra de las líneas que confluirá en la lucha contra las dirigencias constituidas al relanzar luego del paro general del 6 de diciembre de 1982 la histórica Lista Marrón, ahora como "Agrupación 6 de diciembre". Los contactos externos construidos en el exilio proveyeron fondos para la creación de los Centros de Formación Sindical (CEFS) que, desde octubre de 1983, comenzaron a editar un boletín y al año siguiente la revista Democracia Sindical.

Como vemos, a partir de 1983 los diversos intentos de organización comenzaron a cristalizar. Entre el 6 y 7 de agosto de 1983 tuvo lugar el Plenario Nacional de las ASP en Villa Carlos Paz, provincia de Córdoba, con representantes de Mendoza, San Luis, San Juan, Tucumán, Chaco, Santa Fe, Misiones, Córdoba, Formosa, Capital Federal, Conourbano bonaerense y Mar del Plata, conformándose la Coordinadora Nacional Provisoria de las ASP. Dentro del sector se alineaban una serie de organizaciones cuyo objetivo era confrontar en las elecciones sindicales con las definidas como burocracias, agregando a las reivindicaciones sostenidas por los otros sectores la reincorporación de los trabajadores despedidos por causas políticas o gremiales, la democracia sindical, la libertad de todos los presos políticos y gremiales, el retorno de todos los exiliados y la aparición con vida de los 30.000 detenidos-desaparecidos (Chaves, 2015: 240).

Por su parte, los militantes reunidos en torno al CEFS recuperaban como marco identificatorio los programas de La Falda, Huerta Grande y del $1^{\circ}$ de mayo de la CGT de los Argentinos, así como las figuras de Raimundo Ongaro y del dirigente cordobés Agustín Tosco. Ya desde su primer boletín proponian

la activa participación de los trabajadores para lograr una real y garantizada democracia sindical, que en esencia asegure la plenitud del pluralismo ideológico [...] la independencia de los sindicatos de los patrones, del Estado, de los partidos políticos y de los credos religiosos [...] Nuestra propuesta está encaminada en la búsqueda de un proyecto que ponga el acento en la justicia social y en la liberación nacional, que quiebre la dominación y dependencia que somete a nuestro país y a todos los países de Latinoamérica. ${ }^{2}$

2. Boletín del CEFS, octubre de 1983, pp. 4-5. [El subrayado en el original.] 
Me detuve en estos antecedentes porque tanto las publicaciones señaladas como las redes constituidas a su alrededor actuaron como apoyos para los sectores que en el proceso de normalización sindical que se iniciaría en el país disputaron las conducciones con las burocracias, difundiendo a su vez una serie de experiencias desarrolladas en otros países. Por otra parte, como veremos, algunos de estos sectores terminarán confluyendo también en una propuesta política que puso en cuestión el modelo sindical a finales de la década.

\section{El triunfo de Alfonsin y la democratización sindical}

No es mi intención considerar en este apartado la propuesta del gobierno ya analizada en otros trabajos, sino aportar a la reflexión sobre cómo entender la democratización con el objeto de derivar algunos indicadores que nos permitan observar ciertos avances en esa dirección a partir de los procesos emprendidos por algunos gremios.

Hablo de democratización en el sentido propuesto por Tilly, quien la considera como un proceso conflictivo de interacción permanente entre demandantes y contra-demandantes cuyo resultado debe tender a ampliar la participación, disminuir las desigualdades categoriales e impulsar consultas protegidas y vinculantes con relación a las autoridades y a sus políticas públicas (Tilly, 2007: 14). Si aplicamos este concepto al ámbito sindical entiendo que es posible considerar metodológicamente dos dimensiones mutuamente relacionadas: una, referida al funcionamiento interno, que busca aumentar la participación y representación en el lugar de trabajo y en el sindicato, promoviendo acciones tendientes a disminuir las desigualdades de raza, género, entre otras, cuyos indicadores pueden ser los cambios en los estatutos que fortalezcan la representación de los cuerpos colegiados, por ramas, de las seccionales, la incorporación de mujeres a los órganos directivos, entre otros; la competencia entre listas; la inclusión de mecanismos formales e informales de consulta -como el referéndum-e instancias de control sobre las autoridades; así como otros cambios relacionados con el modelo y las prácticas sindicales. La segunda dimensión apunta más a la acción externa, donde el aumento de la participación y la disminución de la desigualdad categorial se ponen de manifiesto en la ampliación del colectivo representado pero no sólo numéricamente sino a partir de la inclusión de categorias de trabajadores tradicionalmente excluidas de las organizaciones sindicales, tales como trabajadores desocupados, precarizados, cuenta propistas, de emprendimientos autogestivos, entre otros. En el mismo sentido se pueden considerar la promoción de cambios en las legislaciones laborales tendientes al reconocimiento del pluralismo y la diversidad de formas organizativas, la integración en 
agrupaciones intersindicales e intersectoriales que planteen objetivos y tomas de posición políticas, trascendiendo las demandas sólo sectoriales, las acciones tendientes a recuperar el componente internacionalista del movimiento sindical propiciando, por ejemplo, redes latinoamericanas.

En base a lo anterior considero que ciertas redes tejidas durante la oposición a las dictaduras sobrevivieron en democracia y sirvieron para repensar el modelo sindical en un sentido democratizador. Trataré de analizar entonces las dimensiones sobre las que se avanzó considerando sólo algunos ejemplos que sirven para informar cuestiones más generales puestas en debate en el marco de la reconstrucción democrática.

¿Cómo plantearon la lucha en el nuevo contexto los sectores sindicales que hemos considerado con anterioridad? Un elemento común fue el de identificar a sus adversarios como burocracias, es decir como estructuras dirigenciales que buscaban mantener el statu quo previo al golpe en lo que refiere a un control monolitico del poder sindical y sobre los afiliados, sin ampliar la participación, reducir las desigualdades ni someterse a la consulta y opinión de sus representados. Eran identificados también con la ortodoxia sindical que anteponia la filiación partidaria peronista a la de clase, buscando restaurar el monopolio del poder que habian detentado en el pasado, sin promover ningún cambio, disputando a su vez una representación corporativa dentro de las filas del propio movimiento peronista (el tercio sindical en la composición de las listas), sin plantear un proyecto político de liberación nacional y latinoamericana ante la difusión creciente de las recetas neoliberales que afectaban a toda la región. Los sectores burocráticos eran asimismo los que podian acomodarse a los planes del gobierno de turno, como intentaba la conducción de las "62" con el gobierno radical, priorizando la negociación de salarios a la recuperación integral de los derechos de los trabajadores. En otras palabras, para las definidas como burocracias, normalizar no era sinónimo de, ni una oportunidad para, la democratización sindical.

A diferencia de esa posición, los sectores que recuperaban tradiciones combativas pretendian utilizar la legalidad abierta para ganar las conducciones en los gremios, con el objeto de promover alternativas organizacionales y políticas, construyendo a su vez una nueva legitimidad a través de la competencia electoral en elecciones limpias, conformando listas plurales con independencia partidaria en defensa de los intereses de clase, propiciando también la organización desde los lugares de trabajo y las instancias de representación colegiada.

Escapa a las posibilidades de este trabajo relevar los resultados de las primeras elecciones sindicales para mostrar los cambios operados en un sentido democratizador. Un trabajo pionero que se ocupó del tema (Gaudio y Domeniconi, 1986) destacó no sólo la importante competencia 
observada sino, además, la presencia de nuevas dirigencias constituidas por sectores jóvenes pero que reivindicaban tradiciones combativas y que consideraban a la democracia como una oportunidad para la democratización. Algunos estudios locales mostrarian también esa tendencia (Rodríguez, 2015). A título de ejemplo vale constatar algunos logros de los sectores que hemos considerado más arriba al acceder a la conducción, por ejemplo en la UOM de Quilmes donde el "Barba" Gutiérrez, militante de la JTP liberado de prisión en diciembre de 1982, constituyó la agrupación Felipe Vallese, lista naranja, triunfando en las elecciones de noviembre de 1984 (Chaves, 2015: 246). También en la seccional de Villa Constitución triunfó la lista marrón de Piccinini; marrón también era la lista de Guillán que triunfó en FOETRA y verde la de Ongaro que ganó en la FGB, defendiendo todas una política de alianzas entre sectores sindicales combativos. ${ }^{3}$ Algunas de las agrupaciones que habian conformado el núcleo fundador de los CEFS constituyeron el Movimiento de Recuperación Sindical, como corriente interna en diferentes sindicatos y seccionales del país; en Mar del Plata, por ejemplo, se formó el Frente de Agrupaciones Sindicales Antiburocráticas (FASA) que sostenía como principios la "unidad, federalismo, democracia, independencia, solidaridad, capacitación, movilización y pluralismo". ${ }^{4}$

El análisis de esos triunfos y de las prácticas específicas que desarrollaron en sus gremios es una tarea aún pendiente, sobre todo si lo que interesa no es el estudio de casos sino considerarlos en conjunto para valorar los logros alcanzados en relación con las tradiciones de las que provenian sus militantes y con los objetivos que se habian planteado en su confrontación con las dictaduras y con las burocracias. Buscando relacionar esas dimensiones: tradición, propuesta, redes de movilización interna y externa y logros en un sentido democratizador, es que me concentraré ahora en la construcción de una estrategia democratizadora tomando como caso a ATE, con la intención de que pueda servir como modelo para avanzar en el análisis de otras experiencias similares.

\section{Los contenidos de la democratización sindical en ATE}

¿Sobre qué dimensiones del concepto de democratización utilizado como marco de referencia avanzaron los sectores que se definian como antiburocráticos y defensores de la democracia sindical? Algunas de

3. Triunfos similares se produjeron en otros gremios y seccionales, cuya enumeración no es posible realizar aquí, con el rasgo común de agrupar a militantes provenientes del peronismo de izquierda, principalmente de la JTP y de diferentes vertientes marxistas. Ver Chaves, 2015; Gordillo, Sangrilli y Rodríguez, 2015, entre otros.

4. Democracia Sindical, año I, n 3, octubre de 1984, p. 2. 
ellas fueron la de sostener la competencia electoral, fortalecer la representación en los lugares de trabajo, incorporar nuevas representaciones como la de las mujeres y sectores juveniles, la capacitación sindical a través de institutos de formación y la integración en redes internacionales. Veremos cómo la nueva conducción de ATE, la lista Verde de ANUSATE, construyó esos contenidos y sobre todo uno que se oponía a la tradición imperante hasta entonces en el sindicalismo peronista: la descentralización para robustecer la representación y participación propiciando también una nueva propuesta política al comenzar la década menemista.

Durante 1983, con el proceso electoral en marcha, la agrupación levantaba la bandera de la "reconstrucción y renovación" de ATE, a la vez que se sumaba a las reivindicaciones comunes sostenidas por el resto del movimiento obrero: normalización sindical de acuerdo con los estatutos anteriores a 1976, plena vigencia de las convenciones colectivas también para los trabajadores estatales y participación gremial en la dirección de las obras sociales. ${ }^{5}$

Paralelamente a esas acciones continuó una intensa tarea de consolidación de apoyos en el interior del país, instando a la formalización de agrupaciones locales que adherian a la nacional. Ya en democracia y una vez aprobado el Reglamento Electoral para las elecciones sindicales en julio de 1984, la tarea militante de la agrupación culminó con la convocatoria al Primer Congreso Nacional de Delegados y Octavo Plenario anual de ANUSATE, reunido el 31 de agosto de 1984, para definir "el mejor programa, la mejor propuesta y los mejores hombres para conducir ATE". ${ }^{6}$ Participaron 482 delegados de 44 seccionales de todo el país que trabajaron durante dos dias en comisiones para elaborar las propuestas programáticas para las diferentes áreas.

En los comicios realizados el 6 de noviembre en 24 seccionales se enfrentaron la lista Verde ANUSATE con la oficialista Azul y Blanca, en 10 se presentó sólo la Verde y en 14 sólo la Azul y Blanca. Entre las seccionales que apoyaron sólo a la Verde se encontraban algunas de las que reunían mayor cantidad de afiliados como eran las de Buenos Aires y Río Turbio. ${ }^{7}$

En la composición de la Lista Verde se dio prioridad a los representantes del interior, tratando de llevar a la práctica el sentido federal declamado. Así, de los quince candidatos a miembros del Secretariado, cinco lo eran de Buenos Aires y los otros diez representaban a las sec-

5. Asociación de Trabajadores del Estado. Agrupación Nacional Unidad y Solidaridad, septiembre-octubre de 1983, p. 11.

6. ANUSATE, s/f, p. 3.

7. Noti-ATE, 15 de octubre de 1984, p. 1. 
cionales de Córdoba, San Martín, Paraná, Posadas, Mendoza, Borghi, Tucumán, Santa Fe, Santa Rosa de La Pampa y San Juan. Lo mismo ocurría con los vocales donde de los titulares ocho eran del interior y dos de Buenos Aires; en los suplentes la relación era de 21 y 5, respectivamente. De los 35 delegados titulares a los congresos de CGT, 29 eran del interior y 6 de Buenos Aires. ${ }^{8}$

La intención de esta estrategia pareciera haber sido la de reeditar los apoyos fundacionales de la organización, cuando ésta se constituyó con un claro sentido federativo en torno a los trabajadores de los talleres de la Dirección Nacional de Puertos a lo largo de los ríos Paraná y Uruguay, para luego extenderse a otros servicios y empresas estatales en Córdoba, San Juan, Mendoza, Comodoro Rivadavia, Río Gallegos. También, coincidiendo con el argumento de Armelino (2010), para reforzar la identidad de clase que se había ido desdibujando en torno a la figura de empleado o servidor público. Otra cuestión a destacar es que la Lista Verde propuso candidatas mujeres para el Consejo Directivo Central en 8 de las 34 seccionales donde se presentó. ${ }^{9}$

Los resultados de la elección del 6 de noviembre de 1984 dieron el triunfo a De Gennaro-Sbarbatti por una diferencia de cerca de 5.400 votos. ${ }^{10}$

La nueva conducción de ATE puso mucho énfasis en replantear el papel del sindicalismo en el nuevo contexto haciendo permanente referencia a un "modelo sindical para la liberación" que, en el plano interno, implicaba ampliar la participación a través de la incorporación "a la militancia social" de jóvenes y mujeres, así como la descentralización para que las seccionales tuvieran mayor participación, objetivo que se plasmó con la reforma de estatutos de 1988. Hacia afuera apoyaba un programa para un acuerdo nacional en base a los 26 puntos de la CGT que congregara a todas las fuerzas económicas, sociales y políticas en un "Congreso para la unidad nacional" y formulara una propuesta en contra de la deuda externa, exigiendo su moratoria y actuando en conjunto con el resto de los países latinoamericanos "que tienen las mismas preocupaciones y los agreden los mismos enemigos". ${ }^{11}$ Esto los llevaba a definir cómo entendían el "sindicalismo moderno", estableciendo un contrapunto con el discurso de modernizar las relaciones laborales lanzado por el gobierno a través de los proyectos de Caro Figueroa, propuestos por el subsecretario de Trabajo en 1986. Sostenían que esos proyectos no proponian la modernización de las relaciones de

8. Boleta de la Lista Verde ANUSATE, elecciones del 6 de noviembre de 1984.

9. Ibídem.

10. Clarín, 12 de noviembre de 1984, p. 14.

11. ANUSATE. Hacia nuestro IX Congreso nacional de delegados, 1987, p. 5. 
explotación, mientras que para ATE el desafio era la instalación de un modelo social y de desarrollo más justo. Por eso sostenían

la democratización de la sociedad en su conjunto, también la democratización y el fortalecimiento de las estructuras del Estado, incluyendo sus empresas y servicios y su administración. Con la necesidad de una imperiosa desburocratización y un reordenamiento funcional, creando espacios de participación permanente, donde los trabajadores estatales a través de sus organizaciones podamos expresar y defender nuestros intereses y realizar aportes y propuestas concretas, buscando siempre que las nuevas características del Estado lo perfilen como herramienta estratégica en un proceso liberador, nacional y popular. ${ }^{12}$

Pero también, como se señaló, esa acción no podía llevarse a cabo sólo nacionalmente, sino que requería actuar en conjunto a través de estructuras de alcance internacional, como por ejemplo de la Confederación Latinoamericana de Trabajadores Estatales (CLATE), a la que adhería. Por eso ATE mantuvo una presencia permanente en los encuentros realizados por ella, desarrollando un importante protagonismo como anfitriona en el VII Congreso que se realizó en Buenos Aires entre el 8 y 10 de septiembre de 1988. Del mismo modo asistió a la Primera Conferencia sobre Privatizaciones, convocada por la CLATE y realizada en San Pablo entre el 14 y 15 de septiembre de $1990 .{ }^{13}$ La relación con el resto del movimiento obrero latinoamericano fue un objetivo permanente de ATE como parte de la estrategia mayor de la CLAT y CMT a las que adhería y dentro de la propuesta de conformar el "Movimiento de los Trabajadores" que lo acercaba al modelo de sindicalismo de movimiento social.

Lo anterior se ligaba también, como ya señalé, con la intención de recuperar la condición de clase por parte de ATE, aspecto que se hacía evidente a través de cómo definian su identidad con expresiones que se reiteraban en su periódico y como slogan del gremio:

No somos "empleados gubernamentales", no somos "servidores públicos", no somos "personal civil”, somos trabajadores, somos los trabajadores del Estado. ${ }^{14}$

12. Ibídem, p. 9.

13. Cuaderno del Instituto de Estudios sobre Estado y Participación (IDEP) de ATE, $\mathrm{n}^{\circ} 7,1990$.

14. ANUSATE. Agrupación Nacional de Unidad y Solidaridad de la Asociación de 
Ese discurso se fue ligando cada vez más a la necesidad de democratizar el Estado, para que verdaderamente sirva como orientador de un proceso de liberación, dado que consideraban que aquél había sido subordinado a los intereses de las corporaciones empresarias, a la vez que el propio gobierno se estaba convirtiendo en portavoz del nuevo consenso neoliberal que gradualmente se iba hegemonizando en la región. Esto se hizo más evidente a medida que comenzaron a plantearse las primeras propuestas privatizadoras, en especial con la llegada de Rodolfo Terragno al Ministerio de Obras Públicas en 1987.

En ese contexto tuvieron lugar las elecciones para la renovación de las conducciones sindicales a fines de agosto de 1988. A nivel nacional se oficializaron dos listas, la Lista Verde y la Lista Naranja, que reunió a sectores de izquierda no peronista. La posición de De Gennaro fue ampliamente respaldada. Esa situación le permitió concretar un objetivo que venía declamando desde 1984: la necesidad de fortalecer la participación y representación de las distintas ramas y regiones que conformaban ATE. Esto se materializó en el $28^{\circ}$ Congreso Extraordinario realizado en diciembre de 1988 cuando se reformó el estatuto. Su definición política quedó plasmada en el art. $3^{\circ}$, inc. K, que señalaba:

Defender el sistema democrático propendiendo a la defensa plena de los derechos humanos y a la democratización del Estado para ponerlo al servicio de la nación. ${ }^{15}$

Pero la innovación fundamental fue la institución de los Consejos Directivos Provinciales (CDP), así como el de Capital Federal y los de los territorios nacionales, que gozarian de autonomía administrativa y gremial en sus actividades y gestiones de carácter provincial, municipal y local, cuyas sedes estarian en las respectivas capitales de esos distritos. Esos consejos serían dirigidos por una comisión administrativa elegida por el voto directo y secreto de los afiliados, siendo su mandato de cuatro años, y por los secretarios generales electos de cada una de las seccionales correspondientes a esos territorios. Lo interesante es que se crearon instancias de deliberación que permitían atender reivindicaciones específicas e incentivaban a la vez la participación de los afiliados. En efecto éstos, a través de la asamblea de afiliados de cada

Trabajadores del Estado, 31 de julio de 1986. Oscar Mengarelli de la seccional Villa Maria era para entonces el secretario general de ANUSATE.

15. Anteproyecto de Reforma de Estatuto de ATE, Buenos Aires, 1 al 3 de diciembre de 1988 , p. 3. 
una de las seccionales, elegían en el mes de marzo de cada año el Congreso Provincial de los CDP, que duraban un año en sus funciones. ${ }^{16}$

Otro mecanismo tendiente a reforzar la participación y democratización fue la introducción del referéndum, que podía ser requerido por el CDN y también solicitado por los CDP, de la Capital Federal o de los territorios nacionales, toda vez que se quisiera someter a deliberación de los afiliados los asuntos que estimaran de interés y dignos de ser tratados en un plano superior, como ser en el Congreso o Consejo Federal.

A su vez, para alentar la participación y formación de los afiliados se crearon los Institutos de Estudios de Estado y Participación (IDEP) y los Institutos de Estudio y Formación (IDEF), para los que se contó también con recursos de redes internacionales.

Además la dirigencia de ATE se preocupó por consolidar otros espacios de reflexión que permitieran generar propuestas para hacer efectiva la democratización tal como la entendían. Ese fue el sentido de su participación activa en el Primer Plenario Nacional de Sindicatos del Movimiento Renovador Peronista, que sesionó en Villa Carlos Paz, provincia de Córdoba, entre el 29 y 30 de mayo de 1986 y que conformó el Movimiento Sindical Renovador Peronista (MSRP). Resulta interesante considerar los principales pronunciamientos de ese sector con relación a cómo entendía la acción gremial, dado que manifestaba un apoyo irrestricto a la unidad de la CGT pero señalaba que en la normalización de la central y de sus delegaciones deberían tenerse en cuenta los criterios de "unidad, libertad, autonomía sindical, democracia, participación y solidaridad", reivindicando la proyección latinoamericana de la CGT. ${ }^{17}$

Lo anterior se liga con otra dimensión de la democratización, la que refiere a su acción externa en pos de un proyecto político más amplio. Pasaré a señalar ahora algunas de las redes construidas en la medida que también incidieron en las tomas de posición que el sindicato fue adoptando.

\section{Estructuras movilizadoras transnacionales en las que participó ATE}

Como señalamos, el apoyo brindado por la CLAT para la formación de ANUSATE fue muy importante y a través de esa central la agrupación entró en contacto con asociaciones ligadas a ella en otros países. Por ejemplo, las vinculaciones con la Asociación Sindical Uruguaya (ASU) la llevó a acompañar y apoyar el proceso que daría lugar al Plenario Inter-

16. Ibidem, art. 49.

17. Documento del I Plenario Nacional Sindical del Movimiento Peronista, Villa Carlos Paz, 29 y 30 de mayo de 1986, p. 12. 
sindical de Trabajadores (PIT) que vio la luz el $1^{\circ}$ de mayo de 1983. Ese día el PIT llevó a cabo la mayor demostración en Uruguay desde 1973, congregando entre 100.000 y 200.000 personas y llamando explícitamente al inmediato retorno a la democracia (Munck, 1989: 141). Sin embargo, a pesar de esa importante movilización, el PIT no fue reconocido por el gobierno en el envío de la delegación uruguaya a la conferencia de la OIT de ese año, por lo que a través del argentino Carlos Custer (ATE), integrante del comité confederal de CMT y del comité ejecutivo de CLAT, se ofreció a dos de sus representantes integrar la delegación de la CMT para participar de esa conferencia y plantear queja contra el gobierno uruguayo (Bottaro, 1985: 137-138).

Dentro de las iniciativas para propiciar acciones conjuntas en pos de la democratización, es de destacar la de ASU de organizar la reunión de trabajadores del Cono Sur en Montevideo en julio de 1983. Alli se reunieron representantes de Brasil, de Chile y Carlos Custer (ATE) por Argentina. ${ }^{18}$

Un nuevo encuentro tuvo lugar ese año, esta vez en Buenos Aires el 16 de noviembre, entre la CGT RA, es decir la conducida por Ubaldini, y representantes del PIT: Toriani, Pereyra, Gomensoro y Read. En conferencia de prensa los uruguayos destacaron que lo ocurrido formaba parte de una política de integración que los había llevado a asistir también al congreso de creación de la CUT en agosto de ese año. La declaración firmada en Buenos Aires proclamaba la voluntad de coordinar esfuerzos para lograr la plena vigencia de las libertades públicas, de los derechos sindicales y de la soberanía nacional y por erradicar políticas económicas inspiradas por los intereses extranjeros y resabios de colonialismo "en el continente de San Martin, Artigas y Bolívar". ${ }^{19}$

Ya antes de la recuperación democrática la CLAT se había manifestado en contra de la explotación en el mundo y en oposición al pago de la deuda externa que soportaban los países de América Latina, para lo que entendía debía actuarse conjuntamente. Con ello seguía una posición similar a la sostenida por la comisión promotora del Movimiento Popular por la Unidad Latinoamericana (MOPUL), electa en octubre de $1983,{ }^{20}$ y de la Universidad de los Trabajadores (UTAL) que promovieron

18. Avanzada, año 3, $\mathrm{n}^{\circ} 16$, agosto de 1983, p. 34.

19. Avanzada, año 3, no 20, diciembre de 1983, p. 45. Gomensoro dijo: "Yo creo que por lo menos en los últimos 30 años no hay antecedentes de la firma de una declaración de este tipo entre trabajadores argentinos y uruguayos".

20. Esa comisión se compuso con representantes de Venezuela, Chile, Costa Rica, Perú, Ecuador, Uruguay, Argentina, también estuvieron presentes el secretario general de la CLAT, el argentino Emilio Máspero, y el secretario adjunto, Eduardo García. Avanzada, año 3, $\mathrm{n}^{\circ} 19$, noviembre de 1983, p. 34. 
la reunión de la "Conferencia Latinoamericana sobre la deuda externa y el futuro de América Latina”, en Lima en diciembre de 1984.

$\mathrm{Al}$ año siguiente, entre el 2 y 3 de mayo, se reunieron en Montevideo representantes del PIT-CNT, CUT, CGT argentina, de centrales de Cuba y de Paraguay (en el exilio), de la CLAT, CMT y de la Federación Sindical Mundial (FSM) para constituir una comisión de trabajo que convocara una conferencia latinoamericana sobre deuda externa. ${ }^{21}$ Entre el 15 y 18 de julio se realizó en La Habana la Conferencia Sindical de Trabajadores de América Latina y el Caribe por la Deuda Externa allí representantes de la CUT propusieron unificar las luchas y fijar el 23 de octubre de 1985 como día internacional de acción continental contra ella. ${ }^{22}$

Nuevamente en marzo de 1986 volvió a reunirse el Consejo de Trabajadores del Cono Sur de la CLAT en Montevideo con la participación de delegados de Argentina (ATE y prensa), Bolivia, Brasil, Chile, Paraguay y Uruguay donde redactaron la Declaración de Montevideo, que destacaba la necesidad de profundizar los procesos democratizadores abiertos, la defensa de los derechos humanos, denunciaba las politicas neoliberales y manifestaba su apoyo a la revolución sandinista, entre otros puntos. ${ }^{23}$

Continuando con esas iniciativas, entre el 24 y 26 de septiembre de 1986 tuvo lugar en Buenos Aires la Segunda Conferencia sobre deuda externa y desarrollo en América Latina y el Caribe. Produjo un documento titulado "Primero el pueblo y después la deuda" y los países del Cono Sur firmaron un documento donde rechazaban las dictaduras todavía vigentes. ${ }^{24}$ Para entonces ya se habian iniciado las tratativas para los acuerdos comerciales entre Argentina y Brasil, por lo que la CUT expresaba su alerta para que lo fueran también de los pueblos, dada la necesidad de "construir una respuesta consistente, fundamentada y unitaria de los trabajadores del Cono sur". ${ }^{25}$

Esas definiciones se complementaban muy bien a su vez con las del IX Congreso de la CLAT reunido en Mar del Plata del 22 al 28 de noviembre de 1987, que condensaron los sentidos acerca del Movimiento de los Trabajadores y de un nuevo orden a construir, pregonados por

21. Boletim Nacional CUT, $\mathrm{n}^{\circ}$ 1, mayo de 1985, p. 7 .

22. Boletim Nacional CUT, $\mathrm{n}^{\circ}$ 2, junio-julio de 1985, p. 14.

23. Avanzada, $\mathrm{n}^{\circ} 45$, abril de 1986, pp. 6-16.

24. La Prensa, 24 de septiembre de 1986, p. 5. Fue firmado por la CGT de Argentina, la COB, la CUT y la CGT de Brasil, el Comando Nacional de Trabajadores y la Central Democrática de Trabajadores de Chile, el MIT de Paraguay y el PIT-CNT de Uruguay.

25. Boletim Nacional CUT, $\mathrm{n}^{\circ}$ 9, agosto-septiembre de 1986, p. 12. Decían: "La lucha contra la deuda externa y la injerencia del FMI está ligada necesariamente a la lucha por un nuevo orden económico internacional sustancialmente diferente al actual que sea resultado de la ruptura con el imperialismo, rumbo a una sociedad socialista", p. 13 [la traducción es mía]. 
esa central desde comienzos de la década. A él asistió la CUT y ASU, de Argentina participaron las organizaciones adheridas al CCAS. El Manifiesto dado es muy representativo de cómo concebían la tarea a desempeñar en relación con los cambios operados en el mundo del trabajo y los desafios de ese presente:

Hoy el desafio más inmediato en el camino de la liberación y de una nueva sociedad es la capacidad del Movimiento de los Trabajadores para generar la alternativa de un Nuevo Desarrollo que inicie aquí y ahora la superación más urgente de la pobreza, de la recesión y del proceso de marginación en marcha. Desarrollo alternativo que dé vigencia concreta a los derechos humanos de los trabajadores que aún tienen empleo, de aquellos a quienes el desempleo y subempleo ha hecho trabajadores informales y cuenta propistas para sobrevivir [...] Un desarrollo distinto [...] en armoniosa preservación de nuestros bienes naturales. Un desarrollo que pasa por la reforma agraria auténtica e integral, por la reforma de la tierra urbana, por las cooperativas y por la autogestión de los trabajadores, por la democratización del Estado, por la articulación e iniciativa de la sociedad civil [...] un nuevo orden universal de paz, justicia, libertad y solidaridad y de efectivo respeto a la dignidad de todos los pueblos. ${ }^{26}$

Estas mismas ideas y lineamientos se reiteraron en el Manifiesto de la CLAT del $1^{\circ}$ de mayo de 1988 y en el documento del IX Congreso de ASU en junio de ese año. ${ }^{27}$ La mayoria de ellos serian también destacados en la Propuesta Politica de los Trabajadores (PPT), gestada en Argentina y que aglutinó a los sindicatos que venían desarrollando una lucha antiburocrática y contra el modelo económico, tales los casos tanto de ATE, del sindicato de prensa y de los gremios que se habían nucleado desde comienzos de los 80 en torno a los CEFS, entre otros.

A partir de 1990 la PPT fue tomando cuerpo. Una serie de encuentros, en mayo, agosto, octubre, fue construyendo la que definieron como "herramienta política de los trabajadores". Se partía del diagnóstico de que la reconversión global del capitalismo había impuesto un plan político también global que obligaba a replantear el papel del sindicalismo para hacer posible la construcción de una alternativa popular con vocación

26. Avanzada, $\mathrm{n}^{\circ}$ 64, diciembre de 1987, pp. 21-30. La cursiva es mía para destacar los principales marcos de sentido compartidos por los sectores sindicales conectados a través de la CLAT.

27. Avanzada, no 70, julio de 1988, pp. 17-36. 
de poder donde se evidenciaba claramente la influencia del modelo del PT brasilero. ${ }^{28}$

Finalmente la forma que adoptó esa herramienta política no fue la de un partido o agrupación política sino la del Congreso de los Trabajadores Argentinos (CTA) conformado en 1992, que desde 1996 pasó a autotitularse Central de los Trabajadores de Argentina, resultando electo Víctor De Gennaro como secretario general. El modelo organizativo y la propuesta política, que recogian el proceso de lucha y la circulación de ideas gestadas durante la década previa, aparecieron para entonces como una alternativa radical al sindicalismo tradicional.

\section{Reflexiones finales}

En el artículo me propuse mostrar que, contrariamente a cierta visión predominante sobre el comportamiento homogéneamente corporativo de los sindicatos durante la reconstrucción democrática, se desarrollaron diferentes propuestas que consideraron a la democracia como oportunidad para la democratización sindical. También destaqué que éstas se nutrieron de experiencias de oposición a las dictaduras y a las conducciones que habían expulsado a los dirigentes que las cuestionaban, como fueron los casos señalados de la FGB, de la UOM de Villa Constitución, de ATE, FOETRA, entre muchos otros.

En todos los casos considerados se pudo apreciar la vinculación con redes transnacionales, ya sea porque sus referentes sufrieron exilios o porque se integraron o contaron con el apoyo de organizaciones de alcance regional o internacional, que actuaron como soportes para la organización de nucleamientos que confrontaron con las dictaduras y luego, ya en democracia, aportaron recursos para la creación de centros, publicaciones y redes que se convirtieron en espacios de socialización politica para sostener estrategias democratizadoras.

Esas redes, locales y con conexiones externas, contribuyeron a definir nuevas agendas y formas de entender la democratización que no quedaba circunscripta a la relación entre bases y dirigentes. En efecto, si bien las reivindicaciones referidas a la consulta interna, a la representación desde los lugares de trabajo, a la defensa del pluralismo ideológico como garantía para la expresión de la identidad de clase, a la ampliación de la participación en la toma de decisiones y, dentro de ella, a la descentralización como estrategia para otorgar mayor visibilidad a las cuestiones locales, se convirtieron en contenidos principales y comunes de cómo entendian la democracia sindical los sectores analizados, también lo es que adosaron otras cuestiones relacionadas con la situación común

28. Documento "El recorrido de la PPT", Buenos Aires, noviembre de 1990. pp. 3-4. 
que atravesaba la región frente a las políticas de ajuste y de reforma del Estado que habían comenzado a implementarse. Esto llevó a recuperar una solidaridad transnacional evidenciada, por ejemplo, en las acciones contra la deuda externa, en el repudio a los gobiernos autoritarios que se mantenían en la región y en la defensa de las democracias como un proceso permanente de expansión de sus límites y no sólo como la recuperación de las instituciones democráticas, tanto en lo que refiere al régimen político como a las instituciones y normativa laboral.

En este sentido el concepto de Tilly sirvió como herramienta para analizar el contenido y dirección de las propuestas, permitiendo ampliar la concepción de la democracia sindical también en el sentido de la elaboración de propuestas políticas que buscaban redefinir la categoría de trabajador y sus formas de representación en el contexto de descomposición de la relación salarial fordista clásica; de ese modo la profundización de la participación y la disminución de la desigualdad categorial no se planteaban sólo hacia el colectivo existente, es decir hacia el interior de la organización sindical, sino que buscaban expandir los limites del mismo.

En efecto, la interacción con otras experiencias internacionales permitió incorporar propuestas que se acercaban bastante a la de sindicalismo de movimiento social, discusión que alcanzaria realce hacia la última década del siglo XX como una de las estrategias posibles de revitalización sindical pero que, sin embargo, ya habían comenzado a ser planteadas en la primera etapa de reconstrucción democrática, en sintonía con los debates que preocupaban al movimiento sindical y a los trabajadores en la región.

\section{Bibliografia}

Armelino, Martín (2010), Reformas de mercado y reacciones sindicales. La experiencia de los trabajadores públicos en Argentina en los años noventa, Buenos Aires, Tesis de Doctorado en Ciencias Sociales, FCS-UBA.

Basualdo, Victoria (2006), "Dictadura militar, sindicalismo combativo y relaciones internacionales: apuntes para una historia reciente de los trabajadores", en Basualdo et al., Antología, a 30 años del golpe, Buenos Aires: CTA, pp.117-138.

Bitrán, Rafael y Alejandro Schneider (1992), "Dinámica social y clase trabajadora durante la dictadura militar de 1976-1983. Estudio de la zona norte del Gran Buenos Aires", en Leoncio Rodríguez et al., Nuevas tendencias en el sindicalismo: Argentina-Brasil, Buenos Aires: BiblosFundación Simón Rodríguez.

Bottaro, José (1985), 25 años de movimiento sindical uruguayo, Montevideo: ASU. 
Chaves, Gonzalo (2015), Rebelde acontecer. Relatos de la resistencia peronista, Buenos Aires: Colihue.

Collier Berins, Ruth (1991), Shaping the political arena. Critical junctures, the labor movement and regime dynamics in Latin America, Nueva Jersey: Princenton University Press.

- (1999), Paths toward democracy. The working class and elites in western Europe and South America, Nueva York: Cambridge University Press.

Falcón, Ricardo (1982), "Conflicto obrero y régimen militar. La resistencia obrera en Argentina (marzo 1976-marzo 1981)", en Bernardo Gallitelli y Andrés Thompson, Sindicalismo y regimenes militares en Argentina $y$ Chile, Amsterdam: CEDLA.

Gaudio, Ricardo y Héctor Domeniconi (1986), "Las primeras elecciones sindicales en la transición democrática", Desarrollo Económico, vol. 26, $\mathrm{n}^{\circ} 103$, octubre-diciembre, pp. 423-454.

Gordillo, Mónica (2013), "Normalización y democratización sindical: repensando los 80", Desarrollo Económico, vol. 53, n 209-210, abril-diciembre, pp. 143-167.

-, Carla Sangrilli y Marina Rodriguez (2015), "Normalizaciones regionales: la CGT Regional Mar del Plata y Córdoba”, en Marcela Ferrari y Mónica Gordillo (comps.), La reconstrucción democrática en clave provincial, 1983-1991, Rosario: Prohistoria, pp. 89-120.

Godio, Julio (comp.) (2003), ¿Un PT en Argentina? Reformular las formas de pensar la politica para entender la experiencia brasileña, Buenos Aires: Corregidor.

Mc Guire, James (1997), Peronism without Perón. Unions, parties and democracy in Argentina, California: Stanford University Press.

Moreira Alves, Maria Helena (1984), Estado e oposição no Brasil (1964-1984), Petropolis: Vozes.

Munck, Ronald (1989), Latin America, the transition to democracy, Londres y Nueva Jersey: Zed Books.

Palomino, Héctor (2005), "Los cambios en el mundo del trabajo y los dilemas sociales", en Juan Suriano, Dictadura y democracia (1976-2001), en Nueva Historia Argentina, tomo X, Buenos Aires: Sudamericana.

Pozzi, Pablo (1988), Oposición obrera a la dictadura, Buenos Aires: Contrapunto.

Rodriguez, Marina (2015), "Normalizaciones sindicales: ¿una oportunidad para la democratización? Un estudio sobre la recomposición sindical en el espacio cordobés, 1984-1988", en prensa.

Tilly, Charles (2007), Democracy, Nueva York: Cambridge University Press.

$$
* * *
$$


Titulo: How to confront union bureaucracies? Some democratization strategies in the 80 s

Resumen: Consideraré algunas estrategias antiburocráticas y democratizadoras planteadas por sectores sindicales que, frente a la salida democrática de 1983, recuperaron tradiciones combativas para enfrentar tanto a la dictadura como a las dirigencias gremiales asociadas con ese pasado, dentro del marco general de revalorización de la legalidad democrática. Presentaré algunos ejemplos de los logros conseguidos por esos sectores en las primeras elecciones sindicales, con el objeto de reflexionar sobre cómo se entendía la democratización sindical en el contexto de los años 80, valorando por último algunos avances en esa dirección. El análisis no pretende una reconstrucción exhaustiva de las experiencias sino ofrecer algunas claves teórico-metodológicas y avances empíricos para pensar tanto la democratización como su contraparte, es decir, la burocratización sindical.

Palabras clave: estrategias antiburocráticas - democratización

\begin{abstract}
I will consider some anti-bureaucratic and democratization strategies proposed by trade union sectors during the 1983 democratic outcome, recovering combative traditions to address the dictatorship and the union leaderships associated with that past, within the general framework of revaluation democratic legality. I will present some examples of these sectors achievements in the first union elections in order to reflect how union democracy was understood in the context of the $80 \mathrm{~s}$, valuing finally some progress in that direction. The analysis does not intend a comprehensive reconstruction of the experiences but to offer some theoretical, methodological and empirical advances keys to discuss union democratization and its counterpart, union bureaucratization.
\end{abstract}

Keywords: anti-bureaocratic strategies - democratization

Recepción: 17 de febrero de 2016. Aprobación: 20 de marzo de 2016. 